\title{
Mentoring in academic medicine: A challenging yet rewarding endeavour
}

\author{
Genc Burazeri ${ }^{1,2^{*}}$ \\ ${ }^{1}$ School of Public Health, University of Medicine \\ Tirana, Albania, ${ }^{2}$ Department of International \\ Health, School for Public Health and Primary Care \\ (CAPHRI), Faculty of Health, Medicine and Life \\ Sciences, Maastricht University, Maastricht, The \\ Netherlands \\ ${ }^{*}$ Corresponding author: \\ gburazeri@yahoo.com \\ Tel.: + 355674077260 \\ Fax.: + 3554363615
}

Received: 18 May 2015

Accepted: 19 May 2015

Key words: Academic Medicine - Education Mentoring

Professional advancement in academic medicine requires development of appropriate abilities for addressing complex and evolving demands of health care delivery and multifaceted service provision in order to improve populations' health. From this point of view, development and acquisition of new and appropriate skills and competencies in academic medicine should be supported by adequately tailored educational programs including mentoring functions as a basic prerequisite for personal growth and professional development.

Based on these considerations, mentoring has been also introduced in academic medicine in order to close the gap between the educational content and the competencies required in practice (1). However, little is currently known about the most effective approaches to mentoring in academic medicine. Mentoring in academic medicine is generally organized around competencies, or predefined abilities, as expected outcomes to increase performance, context-bound productivity and to improve the quality of work in clinical practice and other research and academic tasks (1). In academic medicine, however, mentoring is particularly challenging given the nature and characteristics of the profession.

Current frameworks of mentoring functions have mainly focused on individual factors including intrapersonal characteristics of both mentors and mentees, or interpersonal factors such as mentor-mentee relationships (2). On the other hand, there have been only sparse attempts to properly and comprehensively address external factors which may strongly influence the quality of mentoring relationships. This is mainly due to the scarcity of theoretical models underlying and guiding proper research work consisting of a holistic approach which may help to understand and appreciate the complexity of influences on mentoring, especially the quality of mentoring relationships in academic medicine, an area which includes not only teaching and research activities like the other higher education areas, but also 
health care provision to individual patients and the overall population.

In order to fill this void, the article by Dario Sambunjak seeks to establish an allinclusive conceptual framework of mentoring in academic medicine which incorporates factors operating at individual level (micro level), institutional level (meso level) and societal level (macro level) (3). The main contribution of Sambunjak's work relates to the explicit formulation of external factors including the institutional and societal context of mentoring in academic medicine. Future research should adequately operationalize all these factors and, subsequently, test empirically the overall model in different settings, institutional environments and different cultures (3).

Currently, the evidence on the relationship between mentorship and career choice, career progression, and scholarly productivity in countries of the Western Balkans including Albania is scarce. Particularly, research work in the field of academic medicine is quite obsolete and limited in postcommunist Albania. This is mainly due to the lack of research funds, but also as a consequence of training deficits of researchers and academicians to conduct sound research in line with current international standards and best practices. In this context, mentoring related to research work would be particularly valuable for Albania and other transitional countries in the Western Balkans which face similar difficulties and challenges in the field of academic medicine. In these countries, mentoring would enable mentees access to relevant and precious resources which are basic prerequi- sites for successful applications and grant generation (4). Ultimately, this will enhance productivity of mentees by increasing their performance in general and the quality of research activities in particular. Nevertheless, the effectiveness of mentoring on research productivity, including publication and grant success needs to be formally assessed in countries of South Eastern Europe, as most of the research work on this topic has been conducted predominantly in Western societies (4).

In conclusion, the newly suggested ecological framework by Dario Sambunjak (3) should inform future research especially in transitional countries of South Eastern Europe - a particularly under-researched region to date - addressing adequately the individual, institutional and societal factors related to mentoring in academic medicine.

Conflict of interest: The author declares that he has no conflict of interest.

\section{References}

1. Boudreau JD, Macdonald ME, Steinert Y. Affirming professional identities through an apprenticeship: Insights from a four-year longitudinal case study. Acad Med. 2014;89:1038-45.

2. Janssen S, van Vuuren M, de Jong MDT. Motives to mentor: Self-focused, protege-focused, relationship-focused, organization-focused, and unfocused motives. J Vocat Behav. 2014;85:266-75.

3. Sambunjak D. Understanding wider environmental influences on mentoring: Towards an ecological model of mentoring in academic medicine. Acta Med Acad. 2015;44(1):47-57. DOI: 10.5644/ ama2006-124.126.

4. Sambunjak D, Straus SE, Marušić A. Mentoring in academic medicine: A systematic review. JAMA. 2006;296:1103-15. 